\title{
On factoring of unlimited integers
}

\author{
KAREL HRBACEK
}

\begin{abstract}
Abdelmadjid Boudaoud asked whether every unlimited integer is a sum of a limited integer and a product of two unlimited integers. Assuming Dickson's Conjecture, the answer is negative.

2010 Mathematics Subject Classification 26E35 (primary); 03H05, 03H15, 11A51, $11 \mathrm{U} 10$ (secondary)

Keywords: factoring of integers, nonstandard analysis, unlimited integers, Dickson's conjecture
\end{abstract}

Abdelmadjid Boudaoud $[1,2,3]$ asked whether every large integer is close to a product of two large integers. The question was made precise in the framework of nonstandard analysis by interpreting "large" as unlimited (infinite, nonstandard) and "close to" as having a limited (finite, standard) difference from:

Is every unlimited integer a sum of a limited integer and a product of two unlimited integers?

Symbolically: If $\omega \in \mathbb{Z}$ is unlimited, is

$$
\omega=s+\omega_{1} \cdot \omega_{2}
$$

where $s \in \mathbb{Z}$ is limited and $\omega_{1}, \omega_{2} \in \mathbb{Z}$ are unlimited?

We show that the question has negative answer assuming Dickson's Conjecture about primes in arithmetic progressions. Following [1] we use the internal language of an axiomatic nonstandard set theory such as IST or BST, but the argument works, with minor modifications, in any model-theoretic framework (ultraproducts, superstructures). Only the most basic ideas of nonstandard analysis are required, and those only for understanding of conversion of the problem to an equivalent standard one.

First some simple observations. Let $\omega$ be unlimited and $\pi_{1} \cdot \pi_{2} \cdot \ldots \cdot \pi_{\nu}$ be the prime number decomposition of $|\omega|$. If at least two of the prime numbers are unlimited, or if $\nu$ is unlimited, then clearly $\omega=\omega_{1} \cdot \omega_{2}$ for some unlimited integers $\omega_{1}, \omega_{2}$ and (*) holds with $s=0$. Hence a counterexample to $(*)$ has to have the form $\omega=a \cdot \pi$ where $\pi$ is an unlimited prime number and $a \in \mathbb{Z}$ is limited, $a \neq 0$. Without loss of generality we can assume that $\omega>0$ and $\omega$ is a prime number. Indeed, if every 
unlimited prime number $\pi$ could be expressed in the form $\left(^{*}\right)$ as $\pi=s+\omega_{1} \cdot \omega_{2}$, then $\omega=a \cdot \pi=(a \cdot s)+\left(a \cdot \omega_{1}\right) \cdot \omega_{2}$, which would have the required form $(*)$.

If a prime number $\pi$ is a counterexample to $(*)$, then for each limited $s \in \mathbb{Z}$ there exist an unlimited prime number $\pi_{s}$ and a limited $a_{s} \in \mathbb{Z}, a_{s}>0$, such that

$$
\pi-s=a_{s} \cdot \pi_{s} .
$$

Noticing that $a_{0}=1$ and $\pi=\pi_{0}$, we rewrite $(* *)$ as

$$
a_{s} \cdot \pi_{s}-\pi_{0}=-s .
$$

It follows that, for all limited positive integers $q, r$, the system of Diophantine equations $a_{s} \cdot x_{s}-x_{0}=-s, \quad 0<|s| \leq q$, has a solution where all $x_{s}$ are prime numbers greater than $r$. By Standardization, we can extend the external sequence $\left\langle a_{s}: s \in \mathbb{Z}, s\right.$ limited $\rangle$ to a standard sequence $\left\langle a_{s}: s \in \mathbb{Z}\right\rangle$. By Transfer we deduce that the existence of a counterexample to Boudaoud's question implies the following statement $\mathcal{S}$ of standard number theory:

There is a sequence $\left\langle a_{s}: s \in \mathbb{Z}\right\rangle$ such that $a_{0}=1, a_{s}>0$ for all $s \in \mathbb{Z}$, and for all positive integers $q, r$ the system of Diophantine equations

$$
a_{s} \cdot x_{s}-x_{0}=-s, \quad 0<|s| \leq q
$$

has a solution where all $x_{s},|s| \leq q$, are prime numbers greater than $r$.

On the other hand, if there is a sequence as in the statement $\mathcal{S}$, then there is a standard one, by Transfer. Given such standard sequence $\left\langle a_{s}: s \in \mathbb{Z}\right\rangle$, we can take unlimited $q$ and $r$ and the corresponding solution $\left\langle x_{s}:|s| \leq q\right\rangle$. For each $s$ limited, $a_{s}$ is limited and $x_{s}$ is an unlimited prime number satisfying $x_{0}-s=a_{s} \cdot x_{s}$; so $\omega=x_{0}$ is a counterexample to Boudaoud's question. Therefore it suffices to construct a sequence $\left\langle a_{s}: s \in \mathbb{Z}\right\rangle$ as in $\mathcal{S}$.

The system of equations $\left(S_{q}\right)$ has a solution $\left\langle x_{s}:|s| \leq q\right\rangle$ if and only if the system of congruences

$$
\left(R_{q}\right) \quad x \equiv s \quad \bmod a_{s}, \quad 0<|s| \leq q
$$

has a solution $x_{0}$. The obvious necessary condition for solvability of $\left(R_{q}\right)$ is

$$
\left(a_{s}, a_{t}\right) \mid t-s
$$

for all $0<|s|,|t| \leq q$. It is an easy corollary to the Chinese Remainder Theorem that the condition $(C)$ is also sufficient for the existence of a solution to $\left(R_{q}\right)$; moreover, if $\bar{x}_{0}$ is one solution of $\left(R_{q}\right)$, then every solution is given by $x_{0}(k)=\bar{x}_{0}+A^{q} \cdot k$ where $A^{q}=\left[a_{-q}, \ldots, a_{q}\right]$ is the least common multiple of $a_{s},|s| \leq q$, and $k \in \mathbb{Z}$. We let 
$A_{s}^{q}=A^{q} / a_{s}$; returning to the system $\left(S_{q}\right)$ we see that, assuming the condition $(C)$ is satisfied for all $0<|s|,|t| \leq q$, the system is solvable and all of its solutions are of the form

$$
x_{s}(k)=\bar{x}_{s}+A_{s}^{q} \cdot k, \quad|s| \leq q
$$

where $\left\langle\bar{x}_{s}:|s| \leq q\right\rangle$ is a particular solution of $\left(S_{q}\right)$ and $k \in \mathbb{Z}$. We note that the solutions are given by a set of arithmetic progressions. To obtain the desired result, we need to show that there are solutions where all $x_{s}(k)$ are prime, for arbitrarily large $k$.

Dickson's Conjecture was formulated by Leonard Dickson in [4]:

Let $\ell \geq 1, f_{i}(x)=a_{i}+b_{i} \cdot x$ with $a_{i}$ and $b_{i}$ integers, $b_{i} \geq 1($ for $i=1, \ldots, \ell)$. If there does not exist any integer $n>1$ dividing all the products $\Pi_{i=1}^{\ell} f_{i}(k)$, for every integer $k$, then there exist infinitely many natural numbers $m$ such that all numbers $f_{1}(m), \ldots, f_{\ell}(m)$ are prime.

Dickson's Conjecture implies that in $(F)$ there are arbitrarily large $k$ for which all $x_{s}(k)$, $|s| \leq q$, are prime numbers, provided the following congruence condition is satisfied:

(D) For every prime $p$ there is $k$ such that $p \nmid x_{s}(k)$ holds for all $|s| \leq q$.

So it suffices to show that for every prime $p$ there is a solution $\left\langle x_{s}:|s| \leq q\right\rangle$ of $\left(S_{q}\right)$ such that $p \nmid x_{s}$ holds for all $|s| \leq q$.

The condition $(C)$, which guarantees solvability of $\left(R_{q}\right)$, and therefore of $\left(S_{q}\right)$, does not imply $(D)$ (consider the possibility $a_{0}=a_{1}=1, p=2$ ). We formulate a condition that does, for all $q$.

(E) For every prime number $p$ and every $s \in \mathbb{Z}$ :

If $a_{s}=p^{n} \cdot a_{s}^{\prime}$ with $p \nmid a_{s}^{\prime}$, then there is $r \in \mathbb{Z}$ such that $p^{n+1} \mid a_{r}$ and $r-s=u \cdot p^{n}$ where $0<u<p$.

Lemma 1 If the sequence $\left\langle a_{s}: s \in \mathbb{Z}\right\rangle$ satisfies the conditions ( $C$ ) (for all $s, t \in \mathbb{Z}$ ) and $(E)$, then for every $q>0$ and every prime number $p$ the system $\left(S_{q}\right)$ has a solution $\left\langle x_{s}:|s| \leq q\right\rangle$ such that $p \nmid x_{s}$ holds for all $|s| \leq q$.

Proof We fix $q$ and $p$. Let $n$ be the highest exponent such that $p^{n} \mid a_{s}$ for some $|s| \leq q$. Let $q^{*}=q+p^{n+1}$. Let $\left\langle x_{s}:|s| \leq q^{*}\right\rangle$ be a solution of the system $\left(S_{q^{*}}\right)$. Since the restriction of this solution to $|s| \leq q$ is a solution of $\left(S_{q}\right)$, it suffices to prove that for this solution $p \nmid x_{s}$ holds for all $|s| \leq q$. 
We fix $s$ with $|s| \leq q$, write $a_{s}=p^{n} \cdot a_{s}^{\prime}$ with $p \nmid a_{s}^{\prime}$, take $r$ as in $(E)$, and notice that $|r| \leq q^{*}$. The equation $a_{r} \cdot x_{r}-a_{s} \cdot x_{s}=r-s$ follows from $\left(S_{q^{*}}\right)$. We thus have $p^{n+1} \cdot a_{r}^{\prime} \cdot x_{r}-p^{n} \cdot a_{s}^{\prime} \cdot x_{s}=u \cdot p^{n}$, and after simplifying, $p \cdot a_{r}^{\prime} \cdot x_{r}-a_{s}^{\prime} \cdot x_{s}=u$. If $p \mid x_{s}$, then $p \mid u$, a contradiction with $0<u<p$.

It remains to construct a sequence $\left\langle a_{s}: s \in \mathbb{Z}\right\rangle$ that satisfies $(C)$ and $(E)$. We describe its terms $a_{s}$ by their prime factorization $\Pi p^{n_{p}(s)}$. The basic idea is to space those $a_{s}$ that are divisible by $p^{n}$ exactly $p^{n}$ steps apart. (This can be accomplished for $s \geq 0$ by simply putting $a_{s}=s+1$; however, we need a sequence that has this property and is defined for all $s \in \mathbb{Z}$.)

Definition 2 For $p>2$ we let the anchor

$$
s(p, n)=\left(p^{n}+1\right) / 2
$$

we also let

$$
s(2, n)=\left(1-(-2)^{n}\right) / 3=\Sigma_{i=0}^{n-1}(-2)^{i} .
$$

Lemma 3 If $m<n$, then $s(p, n) \equiv s(p, m) \bmod p^{m}$.

Proof For $p>2$ this follows from $s(p, n)-s(p, m)=\left(p^{n}-p^{m}\right) / 2=p^{m} \cdot\left(p^{n-m}-1\right) / 2$. Also $s(2, n)-s(2, m)=\sum_{i=m}^{n-1}(-2)^{i}= \pm 2^{m} \cdot \sum_{i=0}^{n-m-1}(-2)^{i}$.

Definition 4 For every $s$, we let $n_{p}(s)$ be the highest exponent $n$ for which $s \equiv s(p, n)$ $\bmod p^{n}$. A choice of anchors is admissible if $n_{p}(s)$ exists, for all $p$ and $s$.

We note that, for $p>2, s(p, n)=\left(p^{n}+1\right) / 2>0$ and $s(p, n)-p^{n}=-\left(p^{n}-1\right) / 2<0$. Hence for all $n$ such that $|s|<\left(p^{n}-1\right) / 2$ we have $s \not \equiv s(p, n) \bmod p^{n}$, and $n_{p}(s) \leq \min \left\{n: 2|s| \leq p^{n}\right\}$. Similarly, for all $n$ such that $|s|<\left|1-(-2)^{n-1}\right| / 3$ we have $s \not \equiv s(2, n) \bmod 2^{n}$; hence $n_{2}(s) \leq \min \left\{n: 3|s| \leq 2^{n-1}\right\}$. These observations show that our choice of anchors is admissible. Taking $n=1$ establishes that for $p>2|s|+1$ we have $n_{p}(s)=0$, so the coefficients $a_{s}=\Pi p^{n_{p}(s)}$ are well-defined. A table of $a_{s}$ for $|s| \leq 12$ is computed below.

\begin{tabular}{|c|c|c|c|c|c|c|c|c|c|c|c|c|}
\hline$n$ & -12 & -11 & -10 & -9 & -8 & -7 & -6 & -5 & -4 & -3 & -2 & -1 \\
\hline$a_{n}$ & 5 & $2 \cdot 23$ & $3 \cdot 7$ & $2^{2} \cdot 19$ & 17 & $2 \cdot 3 \cdot 5$ & 13 & $2^{4} \cdot 11$ & $3^{3}$ & $2 \cdot 7$ & 5 & $2^{2} \cdot 3$ \\
\hline
\end{tabular}

\begin{tabular}{|c|c|c|c|c|c|c|c|c|c|c|c|c|c|}
\hline$n$ & 0 & 1 & 2 & 3 & 4 & 5 & 6 & 7 & 8 & 9 & 10 & 11 & 12 \\
\hline$a_{n}$ & 1 & 2 & 3 & $2^{3} \cdot 5$ & 7 & $2 \cdot 3^{2}$ & 11 & $2^{2} \cdot 13$ & $3 \cdot 5$ & $2 \cdot 17$ & 19 & $2^{5} \cdot 3 \cdot 7$ & 23 \\
\hline
\end{tabular}


Lemma 5 The sequence $\left\langle a_{s}: s \in \mathbb{Z}\right\rangle$ satisfies the condition (C) for all $s, t \in \mathbb{Z}$.

Proof Fix $s, t \in \mathbb{Z}$ and a prime number $p$. By definition of $n_{p}(s), s \equiv s\left(p, n_{p}(s)\right)$ $\bmod p^{n_{p}(s)}$, and by definition of $a_{s}, n_{p}(s)$ is the highest exponent $n$ for which $p^{n} \mid a_{s}$. Similarly, $t \equiv s\left(p, n_{p}(t)\right) \bmod p^{n_{p}(t)}$, and $n_{p}(t)$ is the highest exponent $n$ for which $p^{n} \mid a_{t}$. By Lemma 2 , for $m=\min \left(n_{p}(s), n_{p}(t)\right)$ we have $s\left(p, n_{p}(s)\right) \equiv s(p, m) \equiv$ $s\left(p, n_{p}(t)\right) \bmod p^{m}$; hence $s \equiv t \bmod p^{m}$. Thus, for every prime $p$ the highest power of $p$ that divides both $a_{s}$ and $a_{t}$ also divides $t-s$. From this the condition $(C)$, to wit, $\left(a_{s}, a_{t}\right) \mid t-s$, readily follows.

Lemma 6 The sequence $\left\langle a_{s}: s \in \mathbb{Z}\right\rangle$ satisfies the condition $(E)$.

Proof Fix $s \in \mathbb{Z}$ and a prime number $p$ so that $a_{s}=p^{n} \cdot a_{s}^{\prime}$ and $p \nmid a_{s}^{\prime}$; by the construction of $a_{s}, n=n_{p}(s)$. There is a unique $k \in \mathbb{Z}$ such that $s(p, n+1)+k \cdot p^{n+1} \leq$ $s<s(p, n+1)+(k+1) \cdot p^{n+1}$; we let $r=s(p, n+1)+k \cdot p^{n+1}$. Then $p^{n+1} \mid a_{r}$, so in particular $r \neq s$, and $0<s-r<p^{n+1}$. As $s(p, n+1) \equiv s(p, n) \bmod p^{n}$, we have $r \equiv s(p, n) \equiv s \bmod p^{n}$. Hence $s-r=u \cdot p^{n}$ and necessarily $0<u<p$. This proves the condition $(E)$.

We restate the final result in the language of model theory.

Theorem Let $\left({ }^{*} \mathbb{Z},<,+, \times, 0,1\right)$ be an elementary extension of $(\mathbb{Z},<,+, \times, 0,1)$ with $\mathbb{Z} \subset{ }^{*} \mathbb{Z}$. Assuming Dickson's Conjecture, there exist $\omega \in{ }^{*} \mathbb{Z} \backslash \mathbb{Z}$ such that every integer in the galaxy of $\omega$, defined as $\mathbf{G}(\omega)=\{\omega-s \mid s$ limited $\}$, factors as $\omega-s=a_{s} \cdot \pi_{s}$ where $a_{s} \in \mathbb{Z}$ and $\pi_{s}$ is an unlimited prime number. In the example constructed above, the galaxy $\mathbf{G}(\omega)$ contains a unique prime number $\omega=\pi_{0}$.

Proof The sequence $\left\langle a_{s}: s \in \mathbb{Z}\right\rangle$ constructed above is first-order definable in the language of $(\mathbb{Z},<,+, \times, 0,1)$. The elementary extension assumption is sufficient to conclude that there are $q, r \in{ }^{*} \mathbb{Z} \backslash \mathbb{Z}$ for which the system $\left(S_{q}\right)$ has a solution where all $x_{s}$ are prime numbers greater than $r$.

According to the construction, the coefficient $a_{s}$ is divisible by an odd prime $p$ if and only if $s=(p+1) / 2+k \cdot p$ for some $k \in \mathbb{Z}$ if and only if $2 s-1=p \cdot(2 k+1)$ for some $k \in \mathbb{Z}$. Thus at least one such $p$ exists, except when $s=1$ and $s=0$. The coefficient $a_{1}=2$. We conclude that all $\omega_{s}-s=a_{s} \cdot \pi_{s} \in \mathbf{G}(\omega)$ are composite except for $\omega_{0}=\pi_{0}$. 
The proofs that the sequence $\left\langle a_{s}: s \in \mathbb{Z}\right\rangle$ satisfies $(C)$ and $(E)$ go through for every admissible choice of anchors $s(p, n)$. The anchors can be chosen so as to make the galaxy $\mathbf{G}(\omega)$ contain (1) no prime numbers or (2) infinitely many prime numbers.

(1) The following values of $s(p, n)$ guarantee that $a_{s} \neq 1$ holds for all $s \in \mathbb{Z}$, and hence that $\mathbf{G}(\omega)$ contains no prime numbers. Let $p_{1}, p_{2}, \ldots, p_{i}, \ldots$ be the increasing enumeration of odd primes. We set $s(2,0)=1, s(2,1)=0, s(2, n)=2^{n-1}$ for $n>1$; $s\left(p_{i}, 0\right)=1, s\left(p_{2 i}, 1\right)=i, s\left(p_{2 i+1}, 1\right)=-i$, and $s(p, n)=s(p, 1)+p^{n-1}$ for all odd $p$ and all $n>1$. It is easy to verify that this choice of anchors is admissible.

(2) Dickson's Conjecture implies that there exist sequences of prime numbers of the form

$$
\langle c+i \cdot(i+1): 0 \leq i<\ell\rangle
$$

for any $\ell$. For $\ell=2$ they are just the prime twins $\langle c, c+2\rangle$. A remarkable example is the sequence $\langle 41+i \cdot(i+1): 0 \leq i<40\rangle$ of 40 primes. We construct $\mathbf{G}(\omega)$ where $\omega+i \cdot(i+1)$ is a prime number for every $i \in \mathbb{Z}, i \geq 0$. This requires a choice of anchors such that, for every prime number $p, s(p, 1) \not \equiv-i \cdot(i+1) \bmod p$ holds for all $i \geq 0$. As $i \cdot(i+1)$ is even, the requirement is guaranteed for $p=2$ by choosing $s(2,1)=1$. For odd primes $p$ the requirement is equivalent to $(2 i+1)^{2} \not \equiv 1-4 s(p, 1) \bmod p$, and thus holds for all $i \geq 0$ whenever $1-4 s(p, 1)$ is a quadratic nonresidue mod $p$. As there are $(p-1) / 2$ equivalence classes $\bmod p$ of quadratic nonresidues, and hence also of possible values for $s(p, 1)$, we can choose $s(p, 1)$ so that $(p-1) / 4 \leq s(p, 1) \leq(p-1) / 2$. This last condition guarantees that the choice of anchors is admissible [if we also let $s(2, n)=\left(1-(-2)^{n}\right) / 3$ and $s(p, n)=s(p, 1)+p^{n-1}$ for $\left.n>1\right]$.

\section{References}

[1] A Boudaoud, La conjecture de Dickson et classes particulières d'entiers, Ann. Math. Blaise Pascal 13 (2006), 103 - 109; https://doi.org/10.5802/ambp.215

[2] A Boudaoud, Decomposition of terms in Lucas sequences, J. Log. Anal. 1:4 (2009), 1 - 23; https://doi.org/10.4115/jla.2009.1.4

[3] A Boudaoud, D Bellaouar, Representation of integers: A nonclassical point of view, J. Log. Anal. 12:4 (2020) 1\{-31; https://doi.org/10.4115/jla.2020.12.4

[4] L E Dickson, A new extension of Dirichlet's theorem on prime numbers, Messenger of Mathematics 33 (1904), 155 - 161.

Department of Mathematics, City College of CUNY

New York, NY 10031,

khrbacek@icloud.com

Received: 9 October 2019 Revised: 21 October 2020 